\title{
Identification of net groundwater recharge rate using an expert system model: a case study of Pintung plane
}

\author{
L. C. Chang ${ }^{1}$, J. P. Tsai ${ }^{1}$, Y. W. Chen ${ }^{1}$ \& C. J. Chiang ${ }^{2}$ \\ ${ }^{1}$ Department of the Civil Engineering, \\ National Chiao-Tung University, Taiwan \\ ${ }^{2}$ Central Geological Survey, Taiwan
}

\begin{abstract}
Groundwater parameter techniques can be classified into two categories: tried and error methods and auto-calibration methods. Tried and error methods usually are time consuming. Most auto-calibration techniques are optimization techniques which rely on sensitivity analysis or a gradient search, which have increased computational demands with increasing parameter dimension. In addition to run time constraints, the applicability of optimization techniques is restricted by its difficult mathematical set up required in its implementation. This paper develops a rapid parameter calibration model, named RGEIS, using the combination of the expert system model and the groundwater simulation model to reduce the computational time and increase the model applicability. The developed model is applied to identify the net recharge rate, the summation of total recharge and total extraction of the study area, of Pintung plane in southern Taiwan. Pintung plane has an area of $78 \mathrm{~km} \times 30 \mathrm{~km}$ with 3 aquifers and complex geological conditions. The study area is divided into 104 parameter zones. Since the planning horizon is 12 months, the total number of the net recharge rate to be calibrated is $1248(104 \times 12=1,248)$. However, only 2,252 seconds are needed for the net recharge identification. The result shows accuracy and efficiency of the proposed model.
\end{abstract}

Keywords: groundwater modeling, parameter identification, expert system.

\section{Introduction}

Model calibration is typically done by trial and error relying on the expertise of the modeler. This process is very slow as the modeler intelligently selects 
parameter combinations that best suits the model. The trial and error methods used by experts can obtain significantly different results due to their expertise and experience. Some research (Madsen [1]; Chau and Chen [2]) indicated that the expertise transformation is extremely difficult between modelers. Therefore, it is not easy to integrate expertise from different experts for better calibration result using the trial and error methods. Many researchers have applied expert system models to improve the model calibration result (Chau [3]; Kim et al. [4]). In addition to the expert system models, Solomatine et al. [5] discussed a variety of optimization techniques to calibrate model parameters. Most of these optimization techniques are based on sensitivity analysis or gradient search methods which are computationally difficult and slow to solve.

This study develops a groundwater parameter calibration model named RGEIS using a rule-based expert system model. This model transforms the calibration expertise into a set of predefined rules that improve the calibration efficiency and ensure high quality calibration results.

Due to insufficient data, the groundwater recharge is usually unknown and is roughly estimated using indirect information. This model treats net groundwater recharge as a parameter and applies the RGEIS model to identify the net groundwater recharge rate, which is the summation of total recharge and total extraction of the study area. The proposed methodology is applied to Pintung plane at southern Taiwan.

\section{Methodology}

The developed model includes two main parts: a rule-based expert system model and a groundwater simulation model. The expert system model is developed using the CLIPS program and the groundwater simulation model is developed using MODFLOW 2000. In this study, the study area is discretized into a number of zones using Voronoi diagram method. Each zone has one hydraulic conductivity, storage coefficient, and net recharge rate. The model development and the calibration process are as follows.

\subsection{The expert system model}

Knowledge acquisition process is needed to collect necessary information and principles for the model calibration using the expert system model. The collected information is then transformed into rules and inference chains using the obtained principles.

Based on the interviews with experts, the calibration knowledge is quantitatively described. These quantitative descriptions are needed to be transformed into rules. The principles for the transformation are listed below.

(1) The parameters are determined using an iterative process that tunes the net recharge rate with a series of parameter adjustments. Before the parameter adjustment, an initial adjustment of the net recharge rate $\left(\Delta \mathrm{Q}^{0}\right)$ should be artificially given as the adjustment reference. 
(2) The recharge rate adjustment is based on the hydraulic head and the aquifer condition. The difference between hydraulic head and model output defines the flow direction of the groundwater, either recharge or extraction. The aquifer condition defines the restrictions based on the geological reality. For example, if the observed head in an unconfined aquifer is higher than the model output, the extraction rate should be adjusted to be smaller.

(3) After the adjustment direction is determined, either increase (or decrease) the recharge rate or extraction rate, the adjustment quantity $\left(\Delta \mathrm{Q}^{\mathrm{n}}\right)$ should be determined. A comparison between two successive adjustment directions is needed to determine the adjustment quantity. If the two adjustments have the same adjustment directions, the adjustment quantity keeps the same. Otherwise, an oscillation effect is observed and the adjustment quantity should be reduced by $50 \%$.

(4) The adjustment quantity $\left(\Delta Q^{n}\right)$ is reduced iteratively by following the previous steps until it converges to a constant. If this constant does not meet the predefined stop criterion, the adjustment quantity is doubled to increase the speed of iteration for efficient purpose.

The abovementioned four principles are transformed into right rules (Table 1) which are kept in the knowledge base of the expert system model. Figures 1 through 3 show that the inference chain is the forward chaining. The detail description of the rules is shown below.

Table 1: $\quad$ The rules used in the expert system model.

\begin{tabular}{|c|c|}
\hline Rule No. & Description \\
\hline Rule 1 & $\begin{array}{l}\text { Is the adjustment quantity big enough or not? If not, the adjustment } \\
\text { quantity should be increased. For the first iteration, adjustment quantity } \\
\text { is assumed to be big enough (i.e. always yes). }\end{array}$ \\
\hline Rule 2 & Is the zone located in the shallow layer? \\
\hline Rule 3 & Is the net recharge positive? \\
\hline Rule 4 & Is the zone in drained condition? \\
\hline Rule 5 & $\begin{array}{l}\text { Is the observed head larger than the model output? This rule determines } \\
\text { the adjustment direction. }\end{array}$ \\
\hline Rule 6 & Is the adjustment direction the same as the previous iteration? \\
\hline Rule 7 & Is the adjustment quantity equal to or less than $10^{-8} \mathrm{~m} /$ day? \\
\hline Rule 8 & Is the observed head larger than the model output? \\
\hline
\end{tabular}

\subsubsection{Parameter calibration}

Equation (1) shows how the proposed model adjusts the recharge rate (or extraction rate). The estimated recharge rate at the iteration $n$ and $n+1$ are denoted by $\mathrm{Q}^{\mathrm{n}}$ and $\mathrm{Q}^{\mathrm{n}+1}$, respectively. The adjustment direction and the quantity for iteration $\mathrm{n}$ are defined by $\mathrm{I}^{\mathrm{n}}$ and $\Delta \mathrm{Q}^{\mathrm{n}}$, respectively. The value of $\mathrm{Q}^{\mathrm{n}}$ is incorporated into the groundwater simulation model to obtain the model's hydraulic head response to the change in net recharge/extraction. This head is used to calculate the adjustment direction $\left(\mathrm{I}^{\mathrm{n}}\right)$ and the adjustment quantity $\left(\Delta \mathrm{Q}^{\mathrm{n}}\right)$. Equation (1) continues to iterate until the model reach the stop criterion.

$$
Q^{n+1}=Q^{n}+I^{n} \Delta Q^{n} \text { where } I^{n}=1 \text { or }-1
$$




\subsubsection{Adjustment direction}

Eight rules form two inference chains (as shown in figures 1 and 2). Figure 1 shows how the model determines the adjustment direction and figure 2 indicates how the adjustment quantity is defined. In figure 1, the inference chain decides whether or not the adjustment quantity is satisfied using Rule 1 . Rule 1 check the adjustment quantity for controlling the calibration efficiency. For initial adjustment, no check is needed. More details about the efficiency can be found in Section 2.1.4.

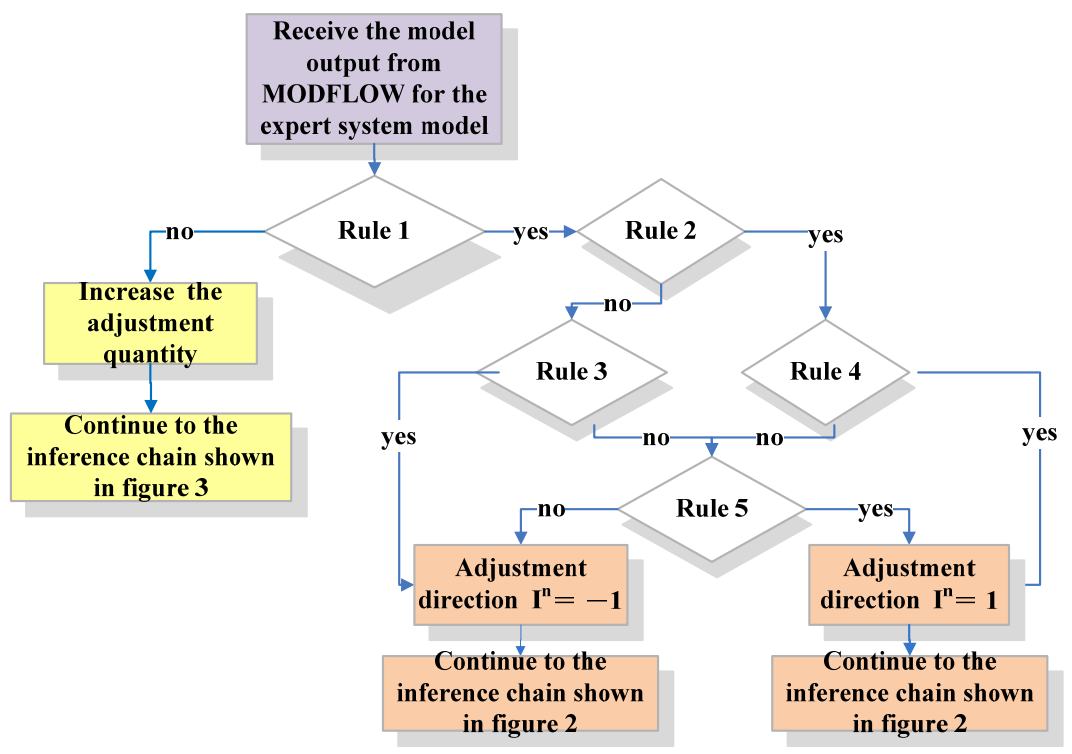

Figure 1: The inference chain of the proposed expert system model for net recharge identification.

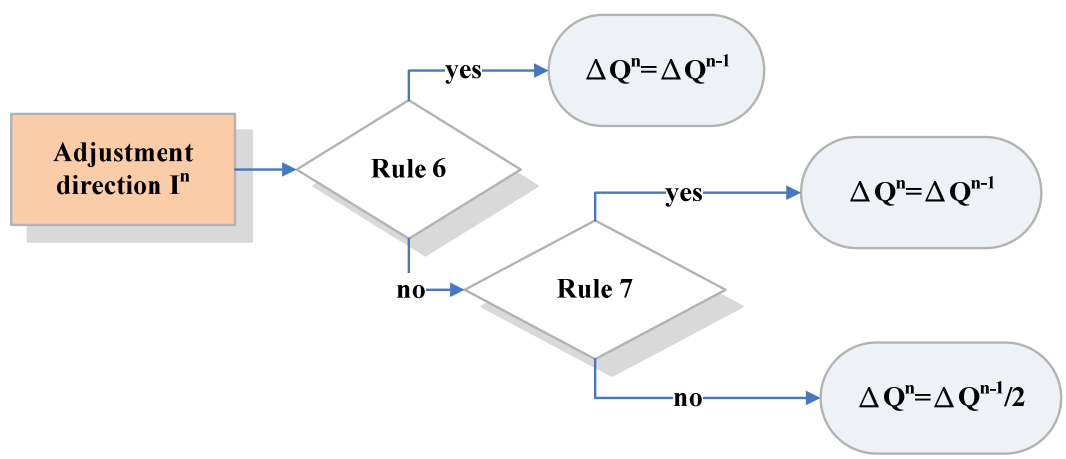

Figure 2: The inference chain for determining the adjustment quantity. 
Rule 2 determines if the zone is in the shallow layer or in one of the deep layers. In this study, different layers have different restrictions on the recharge rate due to its geological conditions. Rule 3 judges the value of the net recharge rate. The positive net recharge rate refers to that water is coming into the zone or the zone is recharging, which cannot be true for zones in deep aquifers because no recharge source is identified in deep layers. If the net recharge rate is positive, we need to change $\mathrm{I}^{\mathrm{n}}$ to be -1 . In the shallow aquifer, Rule 4 is used to identify a zone is drained or not. If the zone is drained, the net recharge should be positive and $\mathrm{I}^{\mathrm{n}}$ should be 1 .

If both Rule 3 and Rule 4 are false, the condition could be either a zone in a shallow aquifer with no drained condition observed or a zone in a deep aquifer under extraction. Under this condition, Rule 5 should be used to change the adjustment direction. If the observed head is larger than the model output, the value of $\mathrm{I}^{\mathrm{n}}$ should be 1 , otherwise, the value of $\mathrm{I}^{\mathrm{n}}$ should be -1 .

\subsubsection{Adjustment quantity}

After the adjustment direction $\left(\mathrm{I}^{\mathrm{n}}\right)$ is decided, the adjustment quantity $\left(\Delta \mathrm{Q}^{\mathrm{n}}\right)$ should be determined (as shown in figure 2). Rule 6 compares the adjustment directions between two iterations. If two adjustment directions are the same, as shown in equation (2), no adjustment is needed. According to Rule 7, if two adjustment directions are the different, as shown in equation (3), the recharge estimation is in an oscillation condition and the adjustment quantity for current iteration $\left(\Delta \mathrm{Q}^{\mathrm{n}}\right)$ should be reduced by $50 \%$ compared to the adjustment quantity for the previous iteration $\left(\Delta \mathrm{Q}^{\mathrm{n}-1}\right)$. However, if the adjustment quantity for the previous iteration $\left(\Delta \mathrm{Q}^{\mathrm{n}-1}\right)$ is smaller than $10^{-2}(\mathrm{~m} /$ day), as shown in equation (4), the adjustment quantity should keep the same value due to the small adjustment.

$$
\begin{gathered}
\Delta Q^{n}=\Delta Q^{n-1} \text {, if } I^{n} \times I^{n-1}>0 \\
\Delta Q^{n}=\Delta Q^{n-1} / 2, \text { if } I^{n} \times I^{n-1}<0 \text { and } \Delta Q^{n-1}>10^{-2} \\
\Delta Q^{n}=\Delta Q^{n-1}, \text { if } I^{n} \times I^{n-1}<0 \text { and } \Delta Q^{n-1}<10^{-2}
\end{gathered}
$$

\subsubsection{Small adjustment}

Equation (5) defines the changing rate of the calibration error, $\eta$, which is used to evaluate the calibration error.

$$
\eta=\left|\frac{\max \left(\vec{\varepsilon}^{n-1}\right)-\max \left(\vec{\varepsilon}^{n}\right)}{\max \left(\vec{\varepsilon}^{n}\right)}\right|
$$

where $\vec{\varepsilon}$ is the calibration error before the iteration $\mathrm{n}$; and $\max \left(\vec{\varepsilon}^{n}\right)$ is the maximum calibration error of all zones. The calibration error is defined as the difference between head observation and model output. The calibration error is a vector $\left(\vec{\varepsilon}^{n}=\left\{\varepsilon_{1}^{n}, \varepsilon_{2}^{n}, \cdots, \varepsilon_{k}^{n}\right\}\right)$. Each element of the vector indicates the calibration error for a zone. 
Rule 1 uses this changing rate of the calibration error to control the calibration efficiency. When $\eta<0.01$ and the calibration process has not completed for all the zones, then the adjustments were reduced at too rapid of a rate resulting in an inefficient calibration. Therefore, the adjustment is tripled for efficiency purpose. The inference procedure is shown in figure 3.

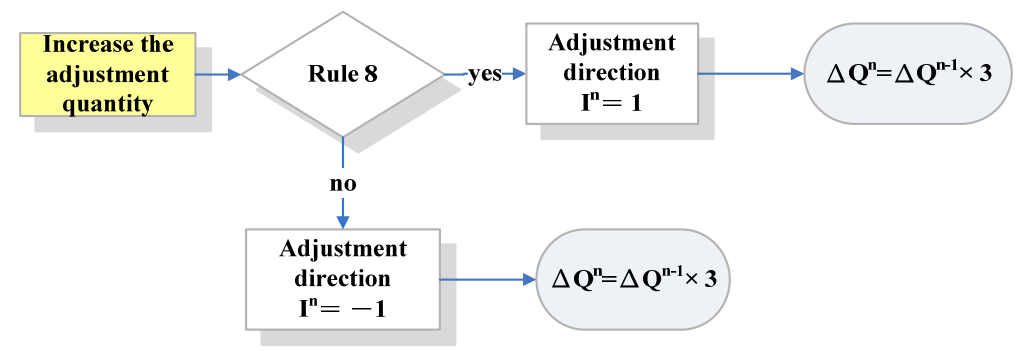

Figure 3: The inference chain for determining the adjustment quantity if the adjustment quantity needs to be increased.

\subsection{Groundwater simulation model of Pintung plane}

\subsubsection{Aquifer structure}

According to the technical report of Central Geological Survey, MOEA [6], the conceptual groundwater structure of the Pintung plane can be shown in figure 4 . Due to insufficient data, layers 6 and 7 are removed from the model. Only layers 1 through 5 are modeled in this study.

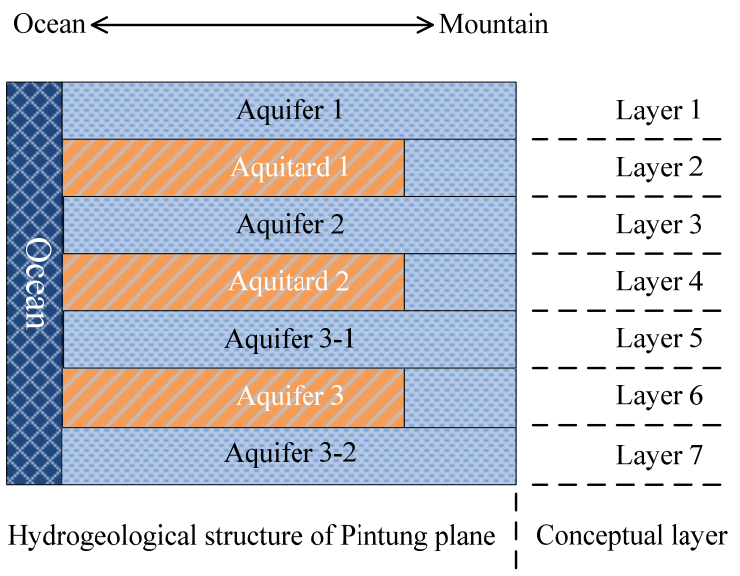

Figure 4: The conceptual hydrogeological structure of Pintung plane. 


\subsubsection{Boundary condition}

The grid size is $1 \mathrm{~km}$ by $1 \mathrm{~km}$. The simulation area is modeled by 78 rows and 30 columns. In the top layer, confined and unconfined conditions are mixed. All other aquifers are confined aquifers. Figure 5 shows the boundary conditions of the model and the grid partitioning.

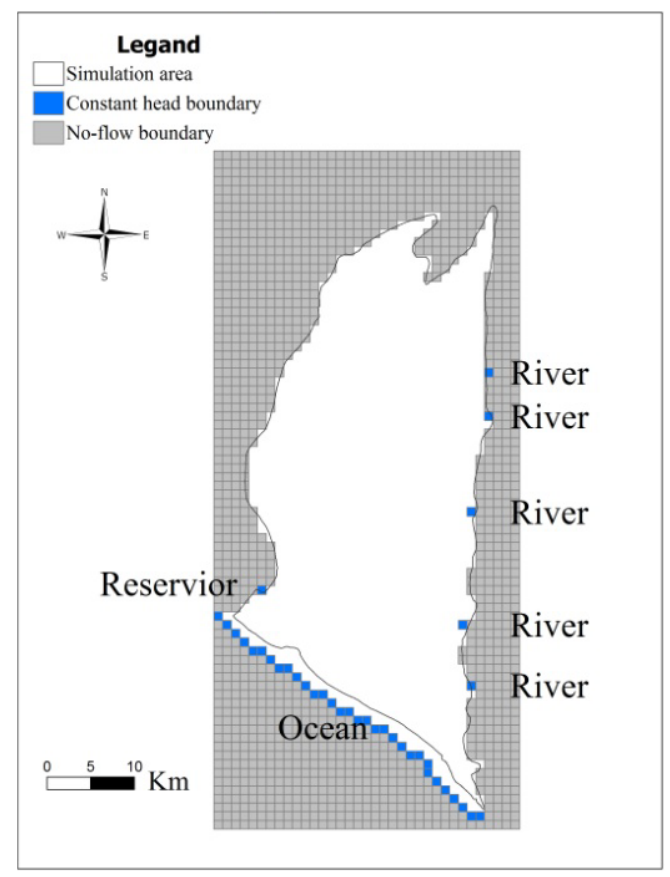

Figure 5: The boundary conditions of Pintung plane.

\subsubsection{Model input}

The main model inputs are time parameters, hydraulic conductivity, storage coefficient, and the initial head.

Time parameters The model's time step is set to 1 day with a stress period of 1 month and planning horizon of 12 months.

Hydraulic conductivity Hydraulic conductivity $(\mathrm{K})$ values are calculated from reported values of transmissivity $(\mathrm{T})$ and aquifer thicknesses. Transmissivity ( $\mathrm{T}$ ) values are reported by Water Resources Agency from drilling data and field experiments at the observation well locations. The obtained hydraulic conductivity is then applied to the entire study area using Thiessen's polygon method. The hydraulic conductivity of the aquitard is defined as $4 \times 10^{-3} \mathrm{~m} / \mathrm{d}$ according to Schwartz and Zhang [7]. The vertical hydraulic conductivity is assumed to be $1 / 10$ the value of the horizontal hydraulic conductivity. 
Storage coefficient Generally, the available data for values of storage coefficient are insufficient. If the storage coefficient is known in a particular well location, this known value is directly assigned to the model for the specific area. If no storage coefficient data are available, then storage coefficient is given using the following rules: (1) an average value is given if the area is in a confined aquifer; and (2) specific yield is given if the area is an unconfined aquifer. The specific yield is calculated based on the aquifer thickness and the material. The specific yield is 0.09 for gravel, 0.12 for coarse sand, and 0.15 for fine sand. If the aquifer is composed of multiple materials, the specific yield is calculated using the weighting method which weights the abovementioned specific yields with their thickness.

Initial head The averaged observed head of December, 1998 is used as the initial head. The head distribution is generated using Kriging method.

\subsection{Parameter calibration process}

Figure 6 shows the flowchart of the parameter calibration process. The initial net recharge rate is given in the MODFLOW simulation model. The proposed expert system model analyzes the calibration error, which is the difference between the head observation and the model output. If the calibration error is smaller than the error tolerance, the calibration process is stopped. Otherwise, iteration for new net recharge rate is needed. The expert system model determines the adjustment quantity of the net recharge rate based on the predefined rules. The obtained adjustment quantity is used to update the estimated net recharge rate. This updated rate is then input in the groundwater simulation model. All abovementioned steps are repeated until the stop criterion is met. This calibration process is applied to each stress period until the entire simulation time frame is completed (i.e. 12 months).

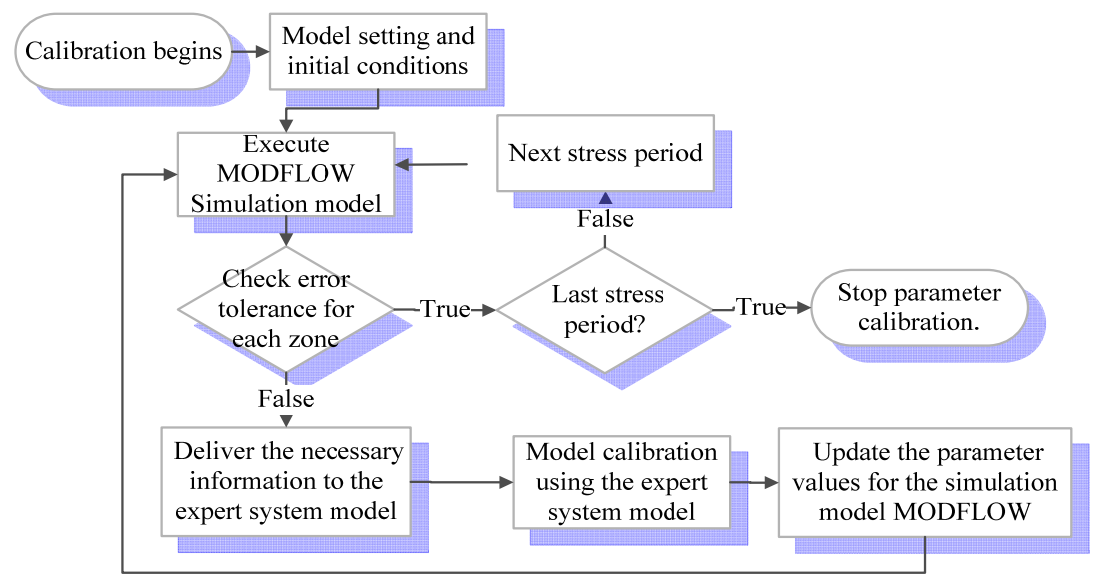

Figure 6: The flowchart of the calibration process for the proposed. 


\section{Case study: Pintung plane}

Pintung plane is divided into 104 parameter zones. Due to large scale and high dimension of parameters, the error tolerance is set to be $2 \mathrm{~m}$ for each zone. The calibration process is a monthly based step-wise calibration. Therefore, the total number of parameters (the net recharge rates) is $1,248 \quad(104 \times 12=1,248)$. According to the geologic condition, the calibrated net recharge rates will be negative for zones in the lower aquifers (extraction condition) and the rate could be positive (recharge condition) or negative (extraction condition) in the top layer.

\subsection{Convergence}

Figure 7 shows the convergence process for January 1999. After 35 iterations, the calibration error is smaller than $2 \mathrm{~m}$ for each zone and the Root Mean Square Error (RMSE) is $0.44 \mathrm{~m}$. The curve of RMSE shows decreasing trend and tend to converge to zero. Note that a RMSE jump can be seen at the iteration No. 32 where the Rule 1 is active and RGEIS triples the adjustment quantity for efficient purpose.

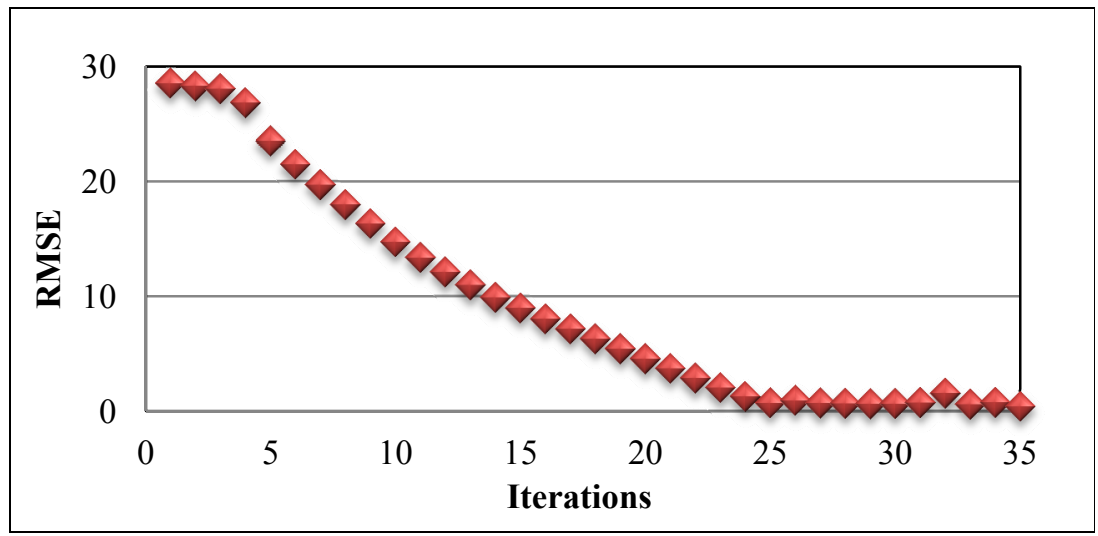

Figure 7: The RMSE changes respect to the hydraulic head versus to the number of iterations.

\subsection{Head distribution in space}

Figure 8 shows the calibration results using contour maps of the hydraulic head. The solid lines indicate the observed head and dashed lines show the model output. The line interval is $5 \mathrm{~m}$. Both observed head and model output show very similar head distribution in the study area. 


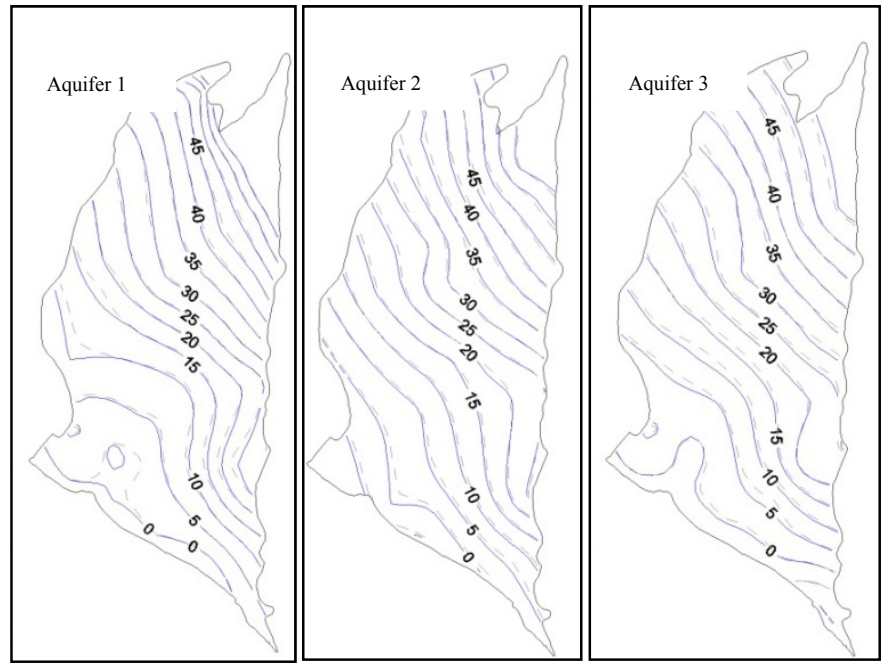

Figure 8: The contour maps of hydraulic head for each aquifer in Pintung plane in 1999.

\subsection{Time series of hydraulic head}

For analyzing the calibration transient results produced by the model. We compare the observed data with the model output at the best-fit location at Dahu observation well in layer 1 (figure 9) and worst-fit location at Chishan observation well in layer 3 (figure 10). The values of RMSE are $0.0007 \mathrm{~m}$ and $1.12 \mathrm{~m}$ for the best-fit location and worst-fit location respectively. Although the RMSE value is relatively high at the worst-fit location, figure 10 shows that the trend is still captured by the calibrated model.

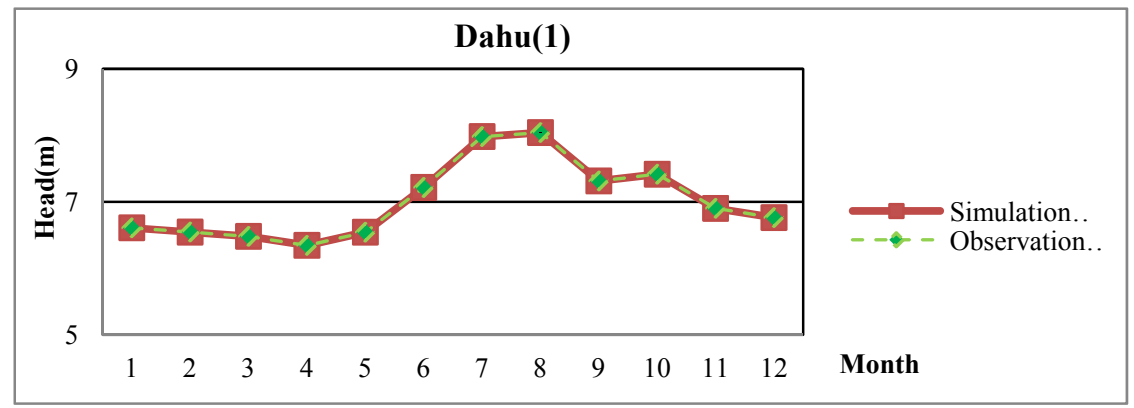

Figure 9: The comparison between the observed head and model output at Dahu observation well in aquifer 1. 


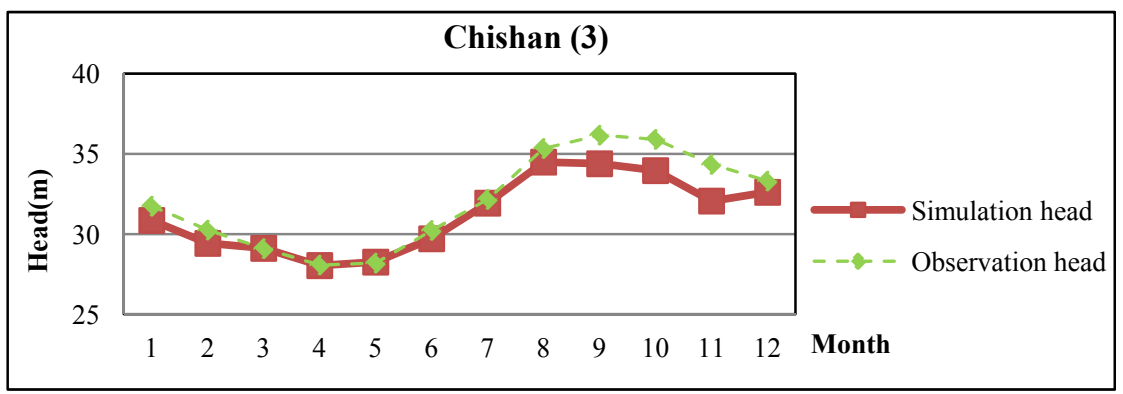

Figure 10: The comparison between the observed head and model output at Chishan observation well in aquifer 3 .

\subsection{Calibration efficiency}

Table 2 shows the number of iterations and elapsed time of the proposed model. The computer uses Intel CPU Core2 Quad 2.66GHz, 4 GB ram, and Fedora 14 as the operation system. In each iteration, RGEIS calls the MODFLOW simulation model once. The calibration for 12 months needs 1,261 iterations and 2,252 seconds. One iteration takes approximately 1.78 seconds (1.12 for simulation model run and 0.67 for expert system model run) to be completed.

Table 2: $\quad$ Number of iterations and time consumption of RGEIS.

\begin{tabular}{|c|c|c|c|c|c|c|c|c|c|c|c|c|}
\hline Month & 1 & 2 & 3 & 4 & 5 & 6 & 7 & 8 & 9 & 10 & 11 & 12 \\
\hline $\begin{array}{c}\text { Cumulated } \\
\text { Iteration } \\
\text { Count }\end{array}$ & 35 & 137 & 295 & 362 & 417 & 579 & 710 & 814 & 874 & 999 & 1073 & 1261 \\
\hline $\begin{array}{c}\text { Elapsed } \\
\text { Time } \\
\text { (second) }\end{array}$ & 62.5 & 244.7 & 526.8 & 646.5 & 744.7 & 1034.0 & 1268.0 & 1453.7 & 1560.9 & 1784.1 & 1916.3 & 2252.1 \\
\hline
\end{tabular}

\section{Conclusion}

This study develops a RGEIS model which combines a groundwater simulation model with an expert system model for net groundwater recharge identification. The developed RGESI model is applied to Pintung plane demonstrating the accuracy and efficiency of the proposed model. The observed head distribution and model results fit well both in time and in space. The Pintung plane has an area of $78 \mathrm{~km} \times 30 \mathrm{~km}$ with 3 aquifers and complex geological conditions. The total number of parameters (the net recharge rates) is 1,248 which took 2,252 seconds to complete the parameter identification. In addition to the high accuracy and efficiency of this method, it is easily to expand it to other models. New rules can be easily added to the expert system model as well as new targets for calibration. This easy expansibility is due to the separation design of control mechanism and data set. 


\section{Reference}

[1] Madsen, H., Automatic calibration of a conceptual rainfall runoff model using multiple objectives. Journal of Hydrology, 235 (3-4), pp. 276-288, 2001.

[2] Chau, K. and Chen, W., An example of expert system on numerical modeling system in coastal processes. Advances in Engineering Software, 32 (9), pp. 695-703, 2001.

[3] Chau, K., A review on integration of artificial intelligence into water quality modeling. Marine pollution bulletin, 52 (7), pp. 726-733, 2006.

[4] Kim, S. M., Benham, B.L., Brannan B, K.M., Zeckoski, R.W. and Doherty, J., Comparison of hydrologic calibration of hspf using automatic and manual methods. Water Resources Research 43:W01402, 2007.

[5] Solomatine, D., Dibike, Y. and Kukuric, N., Automatic calibration of groundwater models using global optimization techniques. Hydrol Sci J, $\mathbf{4 4}$ (6), pp. 879-894, 1999.

[6] Chiang, C.J., Huang, C.C. and Chen, J.E., Hydrogeological investigation and groundwater resources assessment plan in Taiwan, Central Geological Survey, MOEA: Taipei, pp. 101-120, 2002.

[7] Schwartz, F.W. and Zhang, H., Fundamental of Groundwater, John Wiley and Sons, Inc.: New York, pp. 49-52, 2003. 